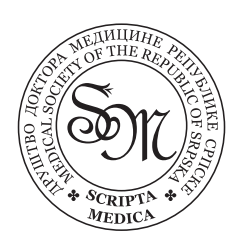

CASE REPORT

\title{
Metastatic Prostate Cancer Presenting as Interstitial Lung Disease
}

\begin{abstract}
Prostate cancer can metastasize to the lungs, but mostly in the form of pulmonary nodules and rarely as lymphangitic carcinomatosis. Also it is rare to have metastatic prostate cancer with no prostatic symptoms at diagnosis. We describe a rare case where a middle aged man presented with isolated pulmonary symptoms and CT scan findings which were consistent with interstitial lung disease, however on subsequent biopsy turned out to be adenocarcinoma arising from the prostate. The symptoms improved with chemotherapy.
\end{abstract}

\section{KEY WORDS}

Prostate, cancer, metastatic, interstitial lung disease, respiratory failure.

\section{Shweta Gupta, ${ }^{1}$ Ozuru Ukoha, ${ }^{2}$ Ashima Sahni, 3 Courtney M.P. Hollowell, 4 Marin Sekosan'}

${ }^{1}$ Div of Oncology, ${ }^{2}$ Div of cardiothoracic Surgery, ${ }^{3}$ Dept of Medicine, ${ }^{4}$ Div of Urology, ${ }^{5}$ Dept of Pathology, John H Stroger Jr. Hospital of Cook County, Chicago, IL 60612, USA

\section{Correspodence}

Marin Sekosan, MD, Chair, Division of Anatomic Pathology, Department of Pathology, John H Stroger Jr. Hospital of Cook County, 1901 W Harrison Street, Chicago Il 6o612, USA Fax: 312-864-9244, Emails: cchmsekosan@yahoo.com,msekosan@ cookcountyhhs.org

Submitted: June 12, 2012

Accepted: September 2O, 2012
A 56-years-old man presented to us with a history of pneumonia and COPD diagnosed May 2010, treated with antibiotics but did not improve to baseline. In November 2010 he was seen at an outside hospital with shortness of breath (SOB), cough productive of yellow phlegm for 10 days. There was no history of sick contacts, recent travel, exposure to respiratory irritants, weight loss, fever, hemoptysis, incarceration or positive PPD. He had a 30-pack year history of smoking cigarettes. Vital signs were normal;Oxygen saturation was $92 \%$ on room air. Systemic exam was remarkable for rhonchi in the chest bilaterally. A CT scan was done which showed bilateral symmetrical extensive interlobular thickening with patchy ground glass opacities of unclear etiology. The pulmonary service was consulted. A presumptive diagnosis of interstitial lung disease was made. He improved with nebulizers, steroids and antibiotics.

The patient was readmitted 3 weeks later at our hospital with worsening shortness of breath so severe that he was unable to walk to the washroom or speak in full sentences. Review of systems was negative. He had no urinary or bowel complaints. He was tachypneic breathing over 22 breaths/minute and oxygen saturation was consistently $<90 \%$ on room air. Systemic exam was remarkable for decreased air entry in the right lung base and dry crackles bilaterally. He was pale and there was bilateral $2+$ pedal edema. The cardiovascular, abdominal and neurologic examinations were unremarkable.
CT scan of the chest (Fig. 1) showed increased interstitial markings and thickening bilaterally, in addition to bilateral small pleural effusions. He hadWBC count of $12,300 / \mathrm{mm}^{3}$ with $79 \%$ neutrophils; hemoglobin was $8.1 \mathrm{~g} / \mathrm{dL}$ and creatinine $1.1 \mathrm{mg} / \mathrm{dL}$.

His condition worsened requiring admission to the Medical Intensive Care Unit because of concern for impending

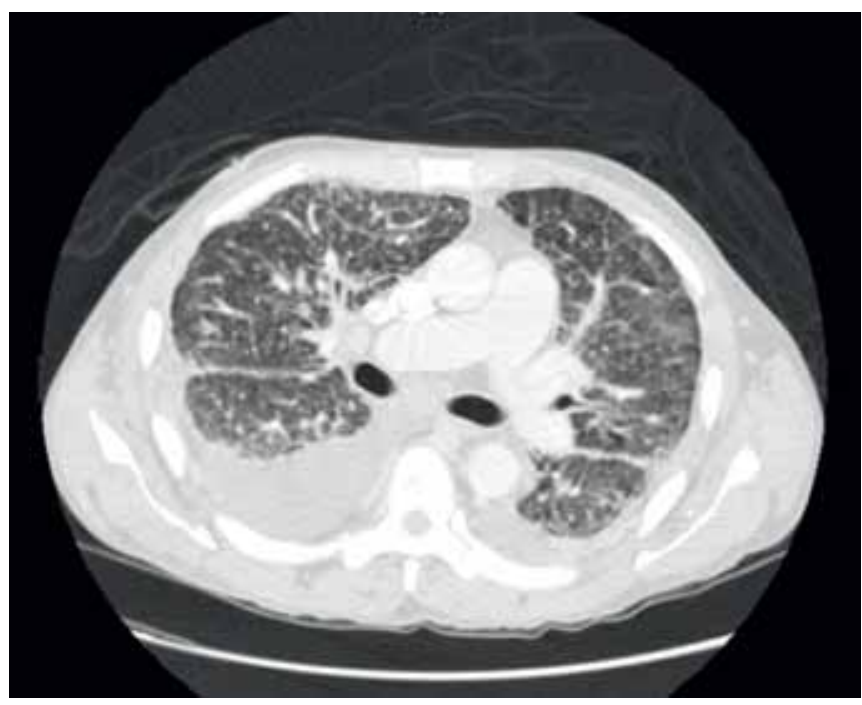

Figure 1. CT scan of the chest at presentation showing increased interstitial markings 


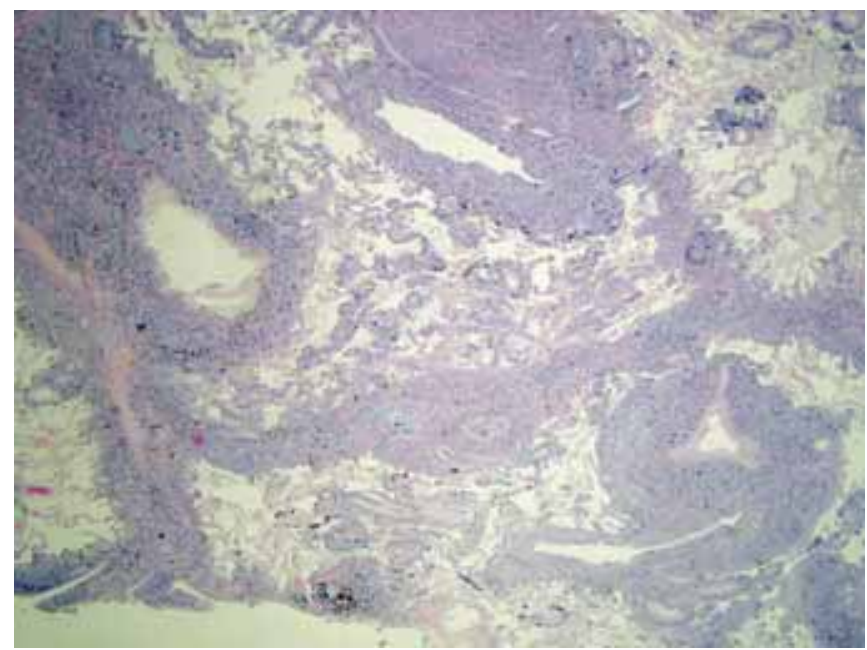

Figure 2. Adenocarcinoma involving bronchilovascular bundles, lymphatics, acinar septae and adjacent alveolar walls $(6.5 \mathrm{X})$

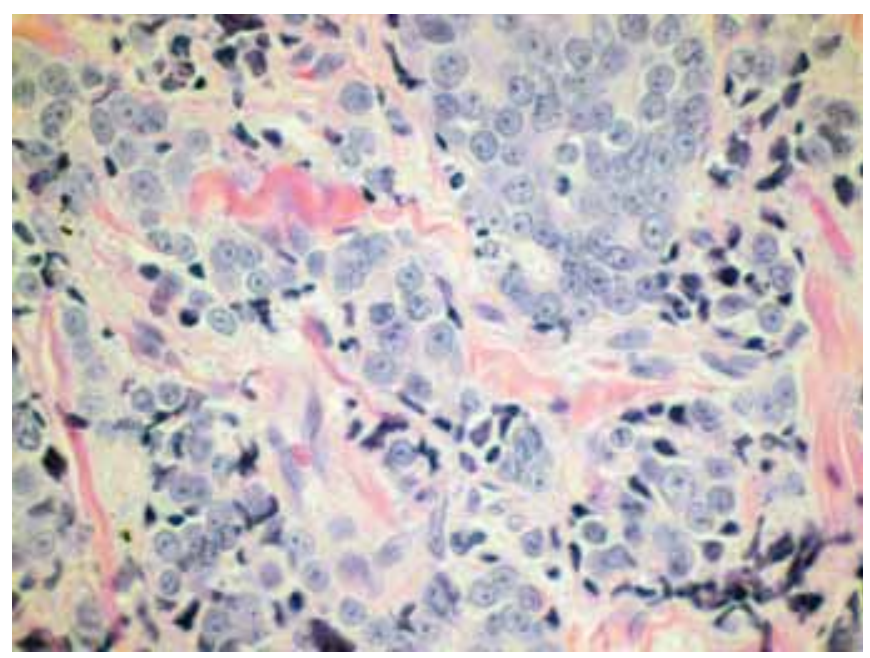

Figure 3. Adenocarcinoma cells with prominent nucleoli (90X)

respiratory failure. Due to an elevated white count and worsening respiratory status, he was empirically started on broad-spectrum antibiotics, consisting of Vancomycin and Piperacillin-Tazobactam, as well as respiratory adjuvants. With his clinical condition deteriorating and no definitive etiology for these parenchymal changes, which were not unlike the more commonly diagnosed pneumonitis or organizing pneumonia, a lung biopsy was requested.

He was taken to the operating room where he underwent left video-assisted thoracoscopic wedge biopsy of the left upper lobe. Grossly the lung tissue was granular, fibrotic and severely thickened such that the staple line had to be reinforced with running chromic suture because of obvious disruption. The parietal pleura was normal and there was $250 \mathrm{ml}$ of straw-colored pleural effusion evacuated from the pleural space. He left the operating room unable to be extubated after the procedure.
Pathology showed adenocarcinoma involving lymphatics, acinar septae, pleura, and bronchiolovascular bundles. In some areas the tumor was also invading into the alveolar walls located adjacent to bronchiolovascular bundles and acinar septae (Fig 2). Tumor cells showed mild variation in size and prominent nucleoli (Fig 3). Tumor cells by immunohistochemistry were strongly positive for prostate specific antigen (PSA), and therefore consistent with prostatic primary. The patient had a serum PSA level of 2864ng/ $\mathrm{ml}$. Subsequent CT scan of the abdomen showed a normal sized prostate with normal abdominal viscera but some suspicious bone lesions.

Clinically, he was unable to be weaned from the ventilator more than one week after surgical biopsy. Medical oncology was consulted and he was given a dose of docetaxel chemotherapy. He subsequently improved quite dramatically such that he was extubated and later discharged on home oxygen. The chemotherapy was continued as an outpatient. He initially refused androgen deprivation therapy but consented to it after cycle 4. His PSA dropped to $116 \mathrm{ng} / \mathrm{ml}$. After 12 cycles of chemotherapy, his CT scan of the chest showed marked improvement. He continues to do well at this time and does not require home oxygen anymore.

\section{Discussion}

The interstitial spread of predominantly adenocarcinomas through the pulmonary lymphatics is commonly referred as pulmonary lymphangitic carcinomatosis (PLC). It constitutes $7 \%$ of pulmonary metastases ${ }^{1}$. The predominant age of presentation reported in the literature varies between 40-49 years. It is commonly seen in neoplasms originating from the breast (33\%), stomach (29\%) and lungs (17\%). ${ }^{2}$ The clinical manifestations of pulmonary lymphangitic carcinomatosis include coughing, panting, dyspnea. A normal or restrictive respiratory pattern could be seen on pulmonary function tests. Lung biopsy is the gold standard for the diagnosis but HRCT has been shown to be equally beneficial in this regard. The common HRCT findings of PLC include thickening of interlobular septa, fissures, and bronchovascular bundles. ${ }^{3}$ The progression once the patient develops PLC is fairly rapid as noted in the literature with mean survival varying between 2-7 months except for prostatic primary carcinoma, which may have good remission with adjuvant hormonal therapy. ${ }^{4}$

It has been described more than two decades ago that pulmonary metastases from prostate adenocarcinoma are found at autopsy in $25 \%$ to $38 \%$ of patients but are evident on chest films in only $5.5 \%$ to $6.7 \%$.

Intra-thoracic involvement from prostate adenocarcinoma could be in the form of lung nodules (84\%), mediastinal lymphadenopathy (12\%) or lymphatic spread $(4 \%)^{5}$. There 
are few case reports of prostate cancer presenting with pleural effusions ${ }^{6}$.Most cases of metastatic carcinoma of the prostate to the lungs have symptomatic prostate primary or bone metastasis as in this case where a left posterior iliac crest biopsy was positive.

Pulmonary involvement with metastatic prostate cancer without bone metastasis has been described in a few case reports ${ }^{2,4,5}$. Interstitial thickening can be a part of the spectrum of radiological findings seen in lymphangitic carcinomatosis. ${ }^{7}$ However, to our knowledge, metastatic prostate cancer presenting as purely interstitial lung disease has not been described.

We have described one of the rare presentations of prostate cancer, which is pulmonary involvement with no prostatic symptoms at diagnosis. Another rarity is the pattern of pulmonary involvement, in this case an interstitial process rather than cannonball lesions. This led to the delay in diagnosis until the patient's respiratory status declined requiring admission to the medical intensive care unit. Our case emphasizes that metastatic prostate cancer to the lungs can take many forms and this differential diagnosis must be considered if an old male patient presents with unexplained new pulmonary findings.

\section{Authorship statement}

OU performed the lung biosy. MS examined the tissue biopsy and made the diagnosis. $S G$ and $A S$ were involed in the oncologic treatment of the patient. CMPH was consulted for his expertise in the field. SG, OU, SG, CMPH and MS contributed to the conception and writing of this article.

\section{Financial disclosure}

The authors declare no conflict of interest.

\section{References}

1. Acikgoz G, Kim SM, Houseni M, Cermik TF, Intenzo CM, Alavi A. Pulmonary lymphangitic carcinomatosis (PLC): spectrum of FDG-PET findings. Clin Nucl Med 2006;31(11):673-8.

2. Bruce DM, Heys SD, Eremin O. Lymphangitis carcinomatosa: a literature review. J R Coll Surg Edinb 1996;41:7-13.

3. Gao Z, Deng X, Cao Z. Pulmonary lymphangitic carcinomatosis. Zhonghua Jie He He Hu Xi Za Zhi. 1998;21(12):739-41.

4. Requena FC, Roma JC, Lleida JV, Blajot I, Gorina AB. Carcinomatous lymphangitis of the lung. Report of 21 cases. Med Clin (Barcelona). 1979;72(6):231-5.

5. Rockey KE, Graham TE. Prostatic adenocarcinoma metastatic to the lung. Postgrad Med 1990;87:199-208.

6. Masue T, Hattori S, Takagi K, Uno M, Komeda H, Fujimoto Y. Prostate cancer detected from pleural effusion: two case reports. Hinyokika Kiyo 2008;54(8):565-8.

7. Karstens JH, Durben G, Ammon J, Frik W, Lindenfelser R. Lymphangitis carcinomatosa of the lung in carcinoma of the prostate: frequency, radiological appearances and clinical significance. Rofo 1979;130(6):629-36. 